\title{
SMOOTH, COMPACT OPERATORS
}

\author{
JULIEN HENNEFELD
}

\begin{abstract}
It is a result of Holub's [Math. Ann. 201 (1973), 157-163], that for $T$ a compact operator on a real Hilbert space, $T$ is smooth $\Leftrightarrow\left\|T x_{1}\right\|=$ $\left\|T x_{2}\right\|=\|T\|$ for some $\left\|x_{1}\right\|=\left\|x_{2}\right\|=1$ implies $x_{1}= \pm x_{2}$. We extend this characterization of smooth, compact operators to a large class of Banach spaces, including $l_{p}, L_{p}[0,1]$, and $d(a, p)$, with $1<p<\infty$. We show that for this same class of Banach spaces, one dimensional, norm one functionals in $K(X)^{*}$ must be extremal. We also present examples of spaces for which Holub's condition does not characterize smooth, compact operators.
\end{abstract}

Introduction. Let $X$ be a Banach space. A norm one element $x \in X$ is called smooth if there exists a unique $f \in X^{*}$ such that $\|f\|=1$ and $f(x)=1$. $K(X)$ and $B(X)$ will denote the spaces of compact operators and all operators on $X$, respectively. A norm one operator is called smooth if it is smooth when considered as a Banach space element.

In Theorem 2.1, we show that if $X$ is assumed only to be a strictly convex, then Holub's condition [3] is satisfied for any smooth operator in $K(X)$ or $B(X)$. In Theorem 2.2, we show that if $X$ is reflexive, smooth, and has a basis, then any compact operator satisfying Holub's condition must be smooth. Also we give examples showing that the hypothesis of compactness cannot be dropped in Theorem 2.2, nor can the hypotheses of reflexivity and smoothness.

Holub's proof of his characterization makes uses of the "polar representation" of a compact operator, which, of course, is only available for Hilbert space. In our proof of Theorem 2.2, we employ instead a much weaker matrix representation for compact operators.

\section{Main results.}

Definition 2.1. $X$ is called strictly convex if each point of the unit sphere is an extreme point.

We now prove the simpler half of the characterization of smooth operators.

THEOREM 2.1. Let $X$ be strictly convex and $T$ a smooth operator in the unit sphere of $B(X)$. If $\left\|T x_{1}\right\|=\left\|T x_{2}\right\|=1$ with $\left\|x_{1}\right\|=\left\|x_{2}\right\|=1$, then $x_{1}= \pm x_{2}$.

Proof. For $i=1,2$ let $y_{i}=T x_{i}$ and let $x_{i}^{*}$ and $y_{i}^{*}$ be of norm one and achieve their norms on $x_{i}$ and $y_{i}$, respectively. Define $f, g \in B(X)^{*}$ by $f(V)=y_{1}^{*}\left(V x_{1}\right)$ and $g(V)=y_{2}^{*}\left(V x_{2}\right)$, for $V \in B(X)$. Then $f=g$ since they

Presented to the Society, January 26, 1979; received by the editors November 28, 1978. AMS (MOS) subject classifications (1970). Primary 47B05; Secondary 46B10, 46C05. Key words and phrases. Compact operator, smooth, reflexive. 
both have norm one and $f(T)=g(T)=1$. Now let $W$ be the operator defined by $W x=x_{1}^{*}(x) y_{1}$, where $x \in X$. Since

$$
f(W)=y_{1}^{*}\left[x_{1}^{*}\left(x_{1}\right) y_{1}\right]=1 \text { and } g(W)=y_{2}^{*}\left[x_{1}^{*}\left(x_{2}\right) y_{1}\right] \text {, }
$$

we must have $\left|x_{1}^{*}\left(x_{2}\right)\right|=1$. Hence, either $x_{1}^{*}\left(x_{1}+x_{2}\right)=2$ or $x_{1}^{*}\left(x_{1}-x_{2}\right)=$ 2. Thus, by strict convexity, $x_{1}= \pm x_{2}$.

Definition 2.2. Let $x, y \in X$. Then $x \perp y$ if $\|x+\lambda y\| \geqslant\|x\|$ for all real $\lambda$. It is well known [2], that in a smooth space, if $f \in X^{*}$ has norm one and achieves its norm on $x$ of norm one, then $\operatorname{ker} f=\{y: x \perp y\}$.

Definition 2.3. A basis $\left\{e_{i}\right\}$ in a Banach space $X$ is a sequence of elements of $X$, such that for each $x \in X$, there is a unique sequence of scalars $\left\{a_{j}\right\}$ such that

$$
\lim _{n \rightarrow \infty}\left\|\sum_{i=1}^{n} a_{i} e_{i}-x\right\|=0 .
$$

TheOREM 2.2. Let $X$ be a reflexive, smooth Banach space with a basis. An operator $T \in K(X)$ of unit norm is smooth if $\left\|T x_{1}\right\|=\left\|T x_{2}\right\|=1$ for some $\left\|x_{1}\right\|=\left\|x_{2}\right\|=1$ implies $x_{1}= \pm x_{2}$.

Proof. First, note that there exists an element of norm one on which $T$ achieves its norm. Otherwise, there would exist a sequence of elements $\left\{x_{n}\right\}$ of norm one such that $\left\|T x_{n}\right\| \rightarrow 1$, and since $X$ is reflexive, we could select a subsequence $x_{n_{k}}$ which converged weakly to some $z \in X$. Then, by the compactness of $T, T x_{n_{k}}$ would converge in the norm topology to $T z$, and thus $T$ would achieve its norm on $z$.

Let $z_{1}$ denote an element of norm one on which $T$ achieves its norm and let $y_{1}=T z_{1}$. It is routine to verify that if a Banach space has a basis, then every closed subspace of co-dimension one also has a basis. Let $z_{1}^{*}$ and $y_{1}^{*}$ be elements in $X^{*}$ of norm one which achieve their norms on $z_{1}$ and $y_{1}$, respectively. Then there exist normalized bases of $X,\left\{z_{i}\right\}_{i=1}^{\infty}$ and $\left\{y_{i}\right\}_{i=1}^{\infty}$, with $\left[z_{2}, z_{3}, \ldots\right]=\operatorname{ker} z_{1}^{*}$ and $\left[y_{2}, y_{3}, \ldots\right]=\operatorname{ker} y_{1}^{*}$.

To each operator $A \in K(X)$ we can associate a matrix with respect to the bases $\left\{z_{i}\right\}$ and $\left\{y_{i}\right\}$. For each $m, n \in N$, let $E_{m n}$ be the operator which sends $z_{n}$ to $y_{m}$ and all other $z_{i}$ to zero. To each $f \in K(X)^{*}$, associate the matrix with $f_{m n}=f\left(E_{m n}\right)$. Maddaus [5] has shown that when $X$ has a basis, each $A \in$ $K(X)$ is the uniform limit of the operators $A_{m}$ with the matrix consisting of the first $m$ rows of $A$ and zeros elsewhere. Since a basis in a reflexive space must be shrinking [4], it follows easily that each $A \in K(X)$ is a limit in the uniform norm of operators formed from $A$ be deleting all but finitely many nonzero entries from the matrix for $A$. Thus, each $f \in K(X)^{*}$ is uniquely determined by its matrix.

Now let $g \in K(X)^{*}$ be the functional which sends $V \in K(X)$ to $y_{1}^{*}\left(V z_{1}\right)$. Since $y_{k} \in \operatorname{ker} y_{1}^{*}$ for $k \geqslant 2$, it follows that the matrix for $g$ has $g_{11}=1$ and all other entries zero.

We will show that if $h \in K(X)^{*}$ achieves its norm on $T$, then $h$ has the 
same matrix as $g$, by considering the following two cases:

Case 1. The matrix for $h$ has a nonzero coordinate outside the first column.

Let us denote the nonzero coordinate $h_{m n}=b$. Let $M$ be twice the basis constant for $\left\{z_{i}\right\}$; then $M$ is a uniform bound on the norms of the coordinate functionals $\left\{\phi_{i}\right\}$ of the basis $\left\{z_{i}\right\}$. Note that

$$
\sup _{\substack{\|x\|=1 \\ \phi_{n}(x)|>| b \mid / M}}\|T x\|=r
$$

is less than 1; otherwise by the reflexivity of $X$ and the compactness of $T$, the fact that $T$ achieves its norm only on $\pm x_{1}$ would be contradicted. Pick $t$ with the same sign as $b$ such that $0<|t|<(1-r) / M$. Let $T_{t}=T+t E_{m n}$ and let $w$ denote an element on which the compact operator $T_{t}$ achieves its norm.

We claim that $\left|\phi_{n}(w)\right|<|b| / M$. Otherwise we would have

$$
\left\|T_{t} w\right\| \leqslant\|T w\|+\left\|t E_{m n} w\right\| \leqslant r+|t M|<r+\frac{(1-r)}{M} M,
$$

and thus $\left\|T_{t} w\right\|$ would be less than 1 , although obviously $\left\|T_{t}\right\| \geqslant 1$.

Finally, observe that $h\left(T_{t}\right)=1+|t b|$, whereas $\left\|T_{t}\right\|<1+|t b|$, since

$$
\begin{aligned}
\left\|T_{t}\right\| & =\left\|T_{t} w\right\|=\|T w\|+\left\|t E_{m n} w\right\| \\
& \leqslant 1+\left|t \phi_{n}(w) y_{m}\right|<1+|t b| / M .
\end{aligned}
$$

Hence, $\|h\|>1$.

Case 2. The only nonzero entries for $h$ are in the first column.

Note that $h_{11}$ must equal 1, otherwise $h(T)$ would not be 1 . To show that $g=h$, it is sufficient to suppose $h_{m 1}=b$ with $b \neq 0$, and $m>1$, and again show $\|h\|>1$.

Since $X$ is smooth, each point in $X$ is Gâteaux differentiable [2], that is

$$
\lim _{t \rightarrow 0} \frac{\|x+t u\|-\|x\|}{t}
$$

exists for each $x, u \in X$. In particular,

$$
\lim _{t \rightarrow 0} \frac{\left\|y_{1}+t y_{m}\right\|-\left\|y_{1}\right\|}{t}
$$

exists, and since $y_{1} \perp y_{m}$, this limit must be zero. Pick $t$ small enough and with the same sign as $b$ such that

$$
\frac{\left\|y_{1}+t y_{m}\right\|-\left\|y_{1}\right\|}{|t|}<|b|,
$$

and let $V_{t}$ be the operator which sends $z_{1}$ to $y_{1}+t y_{m}$. Then $z_{1} \perp z_{k}$ for $k \geqslant 2$ implies that

$$
\left\|V_{t}\right\|=\left\|y_{1}+t y_{m}\right\|<1+|t b|,
$$

whereas $h\left(V_{t}\right)=1+|t b|$.

COROLlaRY 2.1. The Holub characterization of smoothness holds for compact operators on $l_{p}, L_{p}[0,1]$, and $d(a, p)$ with $1<p<\infty$. 
Proof. These spaces satisfy the hypotheses of Theorems 2.1 and 2.2. See [1] and [2].

ExAmple. (a) Let $X$ be as in Theorem 2.2. If $T \in B(X)$ is noncompact, then $T$ need not be smooth even if $T$ satisfies Holub's condition. For example, let $T$ on $l_{p}, 1<p<\infty$ be given by $T e_{1}=e_{1}$ and $T e_{n}=(n /(n+$ 1)) $e_{n}$ for $n>1$. Then $f \in B(X)^{*}$ given by $f(V)=e_{1}^{*}\left(V e_{1}\right)$ where $V \in B(X)$ and $g \in B(X)^{*}$ given by a Banach limit concentrated on the diagonal of $V$, both achieve their norm on $T$.

(b) Let $X=c_{0}$ and $T \in K\left(c_{0}\right)$ have the matrix with first the two rows consisting of $\frac{1}{2}, \frac{1}{4}, \frac{1}{8}, \ldots$ and all other rows consisting of zeros. Then $T$ satisfies Holub's condition and yet $T$ is not smooth.

(c) In the previous example $T$ satisfied Holub's condition by virtue of the fact $T$ did not achieve its norm for any $x$. Now let $X=l_{2} \oplus_{l_{2}} c_{0}$ and let $\left\{d_{i}\right\}$ denote the standard bases of $l_{2}$. Let $T d_{1}=d_{1}, T d_{i}=0$ for all other $i$ and let $T$ act on $c_{0}$ as in the previous example. Then $T$ satisfies Holub's condition by virtue of achieving its norm once.

Proposition 2.1. Let $Y$ be an arbitrary Banach space and $y$ be any smooth element of $Y$. If a norm one element $y^{*} \in Y^{*}$ achieves its norm on $y$, then $y^{*}$ is extremal in the unit ball of $Y^{*}$.

Proof. Suppose $y^{*}=\frac{1}{2}\left(u^{*}+v^{*}\right)$ with $\left\|u^{*}\right\|=\left\|v^{*}\right\|=1$. Then it follows that $u^{*}(y)=v^{*}(y)=1$, and hence $u^{*}=v^{*}=y^{*}$.

We can then conclude that for a large class of Banach spaces one dimensional norm one functionals in $K(X)^{*}$ must be extremal.

Corollary 2.2. Let $X$ be a reflexive, strictly convex, Banach space with a basis. Let $x_{1}$ and $y_{1}^{*}$ be arbitrary norm one elements of $X$ and $X^{*}$, respectively. Then the functional $f \in K(X)^{*}$ defined by $f(A)=y_{1}^{*}\left(A x_{1}\right)$ is extremal.

Proof. Since $X$ is reflexive, $y_{1}^{*}$ achieves its norm on some element which will be denoted by $y_{1}$. Let $\left\{x_{i}\right\}_{i=2}^{\infty}$ be a normalized basis of $x_{1}^{\perp}$, and let $T$ send $x_{1}$ to $y_{1}$ and all other $x_{i}$ to 0 . Then $T$ achieves its norm only once (by strict convexity), and so by Theorem $2.2, T$ is smooth. Finally, note that $f(T)=1$.

\section{BIBLIOGRAPHY}

1. P. G. Casazza and Bor-Luh Lin, Some geometric properties of Lorentz sequence spaces, Rocky Mountain J. Math. 7 (1977), 683-698.

2. J. Diestel, Geometry of Banach space, Lecture Notes in Math., vol. 485, Springer-Verlag, Berlin and New York, 1975.

3. J. R. Holub, On the metric geometry of ideals of operators on Hilbert space, Math. Ann. 201 (1973), 157-163.

4. J. Lindenstrauss and L. Tzafriri, Classical Banach spaces, Lecture Notes in Math., vol. 338, Springer-Verlag, Berlin and New York, 1973.

5. I. Maddaus, On completely continuous linear transformations, Bull. Amer. Math. Soc. 44 (1938), 279-282.

Department of Mathematics, Brooklyn College, Brooklyn, New York 11201 